\title{
BCC: A Novel Block Combination Coding Mechanism
}

\author{
Hai $\mathrm{Fu}^{1, \mathrm{a}}$, Wanrong $\mathrm{Yu}^{2, \mathrm{~b}}$, Chunqing $\mathrm{Wu}^{3, \mathrm{c}}$ and Baokang Zhao ${ }^{4, \mathrm{~d}}$ \\ 1,2,3,4 School of Computer, National University of Defense Technology, Changsha, China 410073 \\ a864853960@qq.com, byu.wanrong@gmail.com, ccqwu@nudt.edu.cn, dbkzhao@nudt.edu.cn
}

Keywords: IPN, NCSTP, Network Coding, Block Combination.

\begin{abstract}
To transmit data reliably and effectively in deep space network, a protocol called NCSTP was proposed, introducing network coding mechanism into space communication. However, the NCSTP cannot make an effective response to the burst packet loss characteristic in InterPlaNetary (IPN) network. Therefore, in this paper we propose a novel mechanism, called block combination coding (BCC). By combining coding blocks in a reasonable manner, this mechanism can effectively reduce the number of retransmission caused by burst packet loss, without increasing coding redundancy. The theoretical analysis shows that, the BCC mechanism can retransmit fewer packets than NCSTP and the simulation analysis also verifies our theoretical analysis results.
\end{abstract}

\section{Introduction}

Deep space link is a typical Delay/Disruption Tolerant Network (DTN) [1,2,3], and there are numerous researches focusing on deep space communication, such as CFDP[4], LTP[5], Saratoga[6], DSTP[7,8], NCSTP[9] and so on. Despite of so many works in this field, how to provide a considerable service in space transmission remains a great challenge, due to the burst packet loss in deep space link.

In this paper, we propose a novel mechanism called block combination coding (BCC). In this mechanism, we combine several coding blocks into a larger combining one, to effectively deal with burst packet loss. With BCC, when burst packet loss happens in a combination block, the receiver side can decode and get the lost packets using the coded redundant packets in each combination block.

The rest of the paper is organized as follows: in Section 2, the related works on transport protocols over the space DTNs are discussed briefly. Section 3 proposes in detail the mechanisms of BCC. Section 4 calculates and determines the block coding coefficient. Section 5 and 6 include the theoretical and simulation evaluation of BCC. Section 7 concludes the paper and provides some directions for future works.

\section{Block Combination Coding Mechanism}

NCSTP introduces network coding mechanism, which is designed to overcome retransmission problems caused by packet loss. It reduces retransmission packets to decrease the transmitting time by forward error correction. However, in deep space networks, due to long transmission distance, the signal reduces severely during transmission and the transmission is always interrupted by unexpected factors, resulting in the packet loss happening in a burst manner, which is called burst packet loss. In this case, the single block coding proposed by NCSTP cannot deal with packet loss effectively. In the rest of this section, we will analyze the probability of burst packet loss through theoretical inference.

According to NCSTP, the number of packets in a coding block is:

$$
N=\frac{1}{P E R}
$$

The probability of i packets lost is:

$$
P=\frac{N !}{i !(N-i) !} P E R^{i}(1-P E R)^{N-i}
$$


Therefore, in this paper, we propose a new mechanism called block combination coding. Through this mechanism, we can deal with the packet burst loss effectively by combining the coding blocks in a reasonable manner without increasing the coding redundancy.

In the single block coding mechanism, it can reach a higher transmission rate by sending a redundant coded packet every $M$ original packets ( $M$ represents $1 / x-1)$. The coded packet is a random linear combination of previous $M$ original packets. In case of one packet lost, the receiver could decode and generate it using the M-1 original packets and the corresponding coded packet. Using the coded packet, NCSTP can generate any original packets if only one packet of this group is lost. If $\mathrm{k}(\mathrm{k}>=2)$ packets in a block are lost, NCSTP needs to retransmit only $\mathrm{k}-1$ packets. Because when these k-1 packets are received, the rest can be generated as well. For example, if $x=0.25$, which means one out of four packets is corrupted on average, NCSTP transmits one coded packet every three original packets (see Fig. 1).

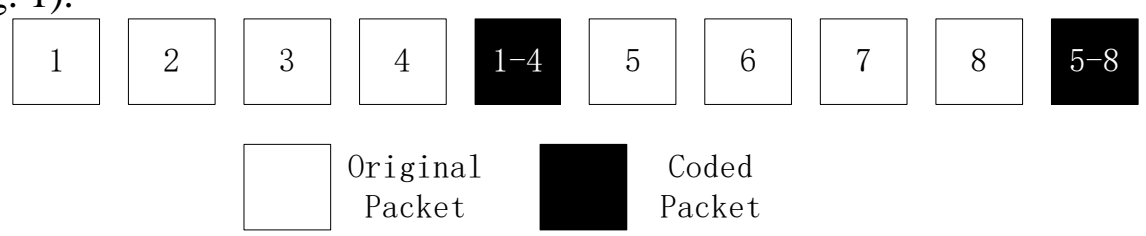

Fig.1 Example of NCSTP packet sequence

According to PER, on average only one packet will be lost in a coding block. So the receiver can decode and generate the lost packet using the coded one. However through our simulation experiment, we find that it happens several times that two packets are lost in one coding block. In real deep space links, the signal is unstable and this phenomenon will be much more common. If accounted with burst packet loss, the performance of NCSTP will decrease apparently.

In the BCC mechanism proposed in this paper, we do not change the original size of coding blocks. Instead, we combine $\mathrm{n}$ (the value of $\mathrm{n}$ will be discussed in next section) coding blocks into a larger combining one and each combining block will generate $n$ linear independent coded packets. In this way, the receiver side can decode and generate the lost packets using the redundant $\mathrm{n}$ coded packets if only the number of lost packets in a combining block is smaller than n. For example, assuming PER is $20 \%$, then in NCSTP there is one coded packet every four original packets. When $n=2$, the BCC mechanism generate 2 coded packets every 8 original packets. So the coding redundancy remains unchanged but it can deal with the case of two packets lost in one combining block. The packet sequence is as shown in Fig. 2.

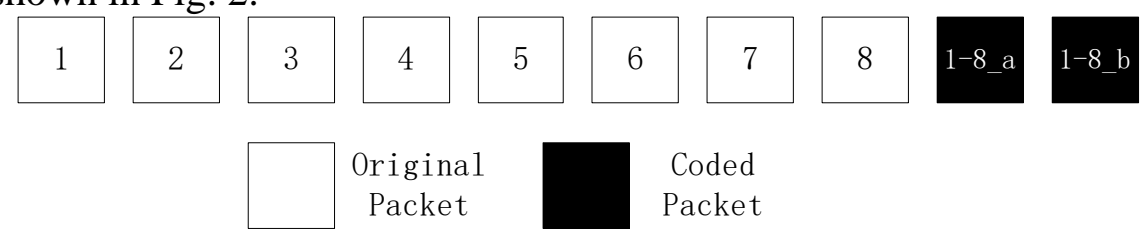

Fig. 2 Example of BCC packet sequence

The most important difference between BCC and dynamically adjusting the size of block is that in BCC, we don't really change the block size. Because we assume that the link PER remains fixed during the whole transmission, so the theoretically optimal block size is settled, which is $1 / \mathrm{x}$ ( $\mathrm{x}$ represents the PER). The BCC mechanism focuses on how many blocks should be combined together to make a larger combining one. Its advantage is that it can cope with burst packet loss without increasing the coding redundancy. As shown in Fig.2, if there are two packets lost in one combining block, then the receiver side can decode the lost two packets with two coded packets of the combining block and needs no retransmission. However in single block coding mechanism, it cannot decode the lost packets and needs at least one retransmission.

\section{Block Combination Coefficient}

In BCC mechanism, we effectively cope with burst packet loss phenomenon by combining $\mathrm{n}$ coding blocks into a larger one. $\mathrm{n}=0$ indicates that there is no coded packet in the sending sequence, while $n=1$ is the single coding block mechanism (NCSTP). However the value of $n$ is not the larger 
the better, because with the increase of $n$, codec complexity increases correspondingly. Therefore, how to set a reasonable value to $\mathrm{n}$, minimizing the probability of packet loss retransmission without increasing the codec complexity greatly, this is a problem needs to be resolved in this section.

In this section, we will determine the specific value of the block combination coefficient $n$ by theoretical analysis. First of all, in order to facilitate the presentation and reading, we will show the variables that will be used in this section and the next section in Table 1.

Table 1 Variables and meaning

\begin{tabular}{|c|c|}
\hline Variables & Meanings \\
\hline$N_{\text {origin }}$ & Number of original packets \\
\hline$N_{\text {block }}$ & Number of packets in a combining block \\
\hline$M$ & Size of coded block in NCSTP \\
\hline$N$ & Packet error rate \\
\hline$P E R$ & Number of blocks in a combining block \\
\hline$n$ & Probability of on retransmission \\
\hline$P_{\text {noretr }}$ & Probability of retransmitting ${ }^{i-1}$ packets in one coding block \\
\hline$Q_{i}$ & Probability of retransmitting ${ }^{i-1}$ packets in one combining block \\
\hline$P_{i}$ & Expectation of retransmission in one coding block in NCSTP \\
\hline$E_{\text {ncrtp }}$ & Expectation of retransmission in one combining block \\
\hline$E$ &
\end{tabular}

According to NCSTP, the number of original packets in a coding block is:

$$
M=\frac{1}{P E R}-1
$$

After combing the coding blocks, the total number of coding packets remains unchanged, $\mathrm{n}$ is number of blocks combined together:

$$
\frac{N_{\text {origin }}}{\left(\frac{1}{P E R}-1\right) \times n} \times n=\frac{N_{\text {origin }}}{\frac{1}{P E R}-1}
$$

The size of combining block is:

$$
N_{\text {block }}=\frac{n}{P E R}
$$

The probability of that the number of loss packets being smaller than $\mathrm{n}$ and requiring no retransmission is:

$$
P_{\text {noretr }}=\sum_{i=0}^{n} \frac{N_{\text {block }} !}{i !\left(N_{\text {block }}-i\right) !} P E^{i}(1-P E R)^{N_{\text {block }}-i}
$$

To calculate the optimal value of $n$, we need to set a threshold, which will be discussed in future work. For example, the threshold is $95 \%$. Then as the $\mathrm{P}_{-}$noretr grows with the value of $\mathrm{n}$, when P_noretr reaches to $95 \%$, we can decide that this value of $\mathrm{n}$ is the optimal value of block combination coefficient. Assuming that PER is 0.01 , then when $n=3$, $P \_$noretr is $98 \%$, larger than $95 \%$.

\section{Theoretical Evaluation}

In order to simplify the theoretical evaluation, some modifications are made to the BCC. We assume that link burst packet loss are purely random, without affection from other factors like signal reduction. We also make assumptions that the link PER remains fixed during the file transmission and ACKs are sent back to the sender side without lost, which means that all acknowledgement information are sent back to the source side successfully. 
In contrast, we firstly analyze the performance of NCSTP without BCC mechanism. In the original NCSTP protocol, the size of coding block is:

$$
N=\frac{1}{P E R}
$$

The probability of losing i packets in one coding block (that is to retransmit i-1 packets) is:

$$
Q_{i}=\frac{N !}{i n(N-i) !} P E R^{i}(1-P E R)^{N-i}
$$

The expectation of the number of packets that need to be retransmitted in one coding block is:

$$
\begin{aligned}
& E_{\text {nertp }}=\sum_{i=2}^{N}(i-1) \frac{N !}{i !(N-i) !} P^{i} R^{i}(1-P E R)^{N-i}
\end{aligned}
$$

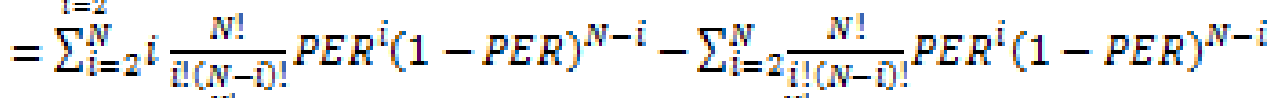

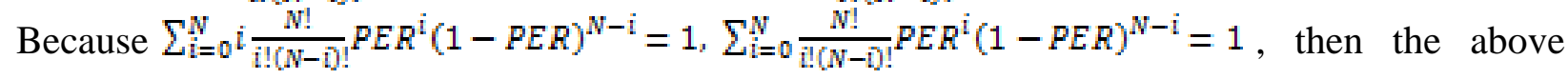
equation simplifies to:

$$
\begin{aligned}
E_{\text {nertp }} & =1-(1-P E R) \frac{1}{P E R}-1 \\
& =(1-P E R)^{\frac{1}{\text { VER }}}
\end{aligned}
$$

With the BCC mechanism, the probability of retransmitting i-n packets in one combination block is:

$$
P_{i}=\frac{N_{\text {block }} !}{i !\left(N_{\text {block }}-i\right) !} P E R^{i}(1-P E R)^{N_{\text {block }}-i}
$$

The expectation of the number of retransmission packets in one combination block is:

$$
\mathrm{E}=\sum_{i=n+1}^{N_{\text {block }}}(i-n) P_{i}
$$

The above equation simplifies to:

$$
E=\sum_{i=0}^{n}(n-i) \frac{N_{\text {block }} !}{i !\left(N_{\text {block }}-i\right) !} P E R^{i}(1-P E R)^{N_{\text {block }}-i}
$$

We can see that when PER is fixed, the number of retransmission packets is a variable related to the block combination coefficient $n$.

Now we evaluate the enhancement of the transmission performance of BCC mechanism by three figures. To show the evaluation more precisely, we assume that the number of original packets $N_{\text {origin }}=100$. Then we set the average number of retransmission ave $=E * N_{\text {origin }} / N_{\text {block }}$. The results of PER=0.1 are shown in the table and figure as follows:

Table 2 Results of PER $=0.1$

\begin{tabular}{|c|c|c|c|c|c|}
\hline$n$ & 1 & 2 & 3 & 4 & 5 \\
\hline ave & 3.4868 & 2.5666 & 2.1248 & 1.8530 & 1.6643 \\
\hline
\end{tabular}

Results in Fig. 3 reveal that, when $n=1$ (NCSTP), 3.4868 packets are needed to be retransmitted on average. However, when $n=2$ (BCC), the result drops to 2.5666, which is a great progress considering the huge retransmission cost in deep space communication. 


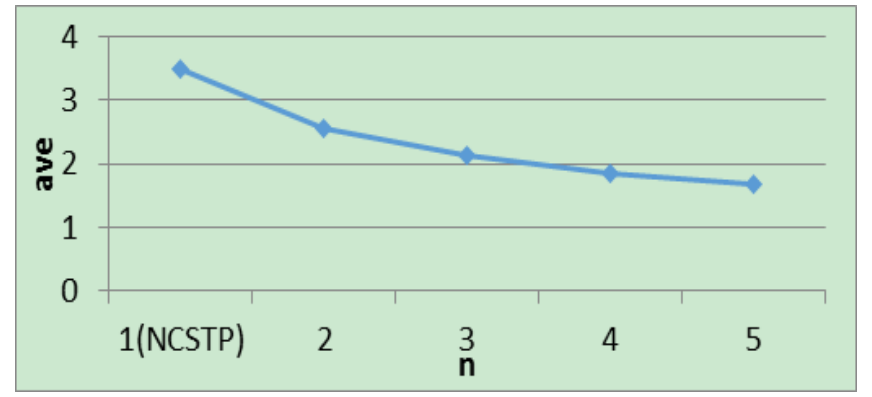

Fig. 3 Result of PER=0.1

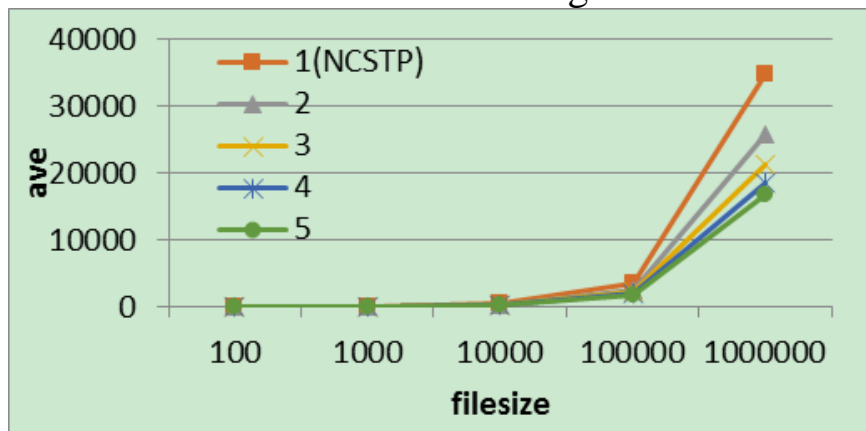

Fig. 5 Retransmission of different filesizes

Fig.4 shows that, the number of retransmission packets in NCSTP grows much faster than that of BCC with bigger PER and larger file size, which means that in deep space networks with high PER, BCC will achieve better performance than NCSTP in transmitting large files.

\section{Simulation Evaluation}

To test and verify our theoretical evaluation, we implement the block combination mechanism in $\mathrm{C}++$ language and explore its performance over a simple one-hop topology. To simplify the simulation, we assume that the link error rate is fixed during our experiment. We run the simulation 100 times and calculate the average number of retransmissions for NCSTP with and without our BCC.

Table 2 Simulation parameter

\begin{tabular}{|c|c|}
\hline Parameter & Value \\
\hline File size (number of packets) & $100,1000,104,105,106$ \\
\hline Packet Error Rate & $0.01,0.05,0.1,0.2,0.25$ \\
\hline Block coding coefficient & $1,2,3,4,5$ \\
\hline
\end{tabular}

With the simulating $\mathrm{C}++$ program that mentioned earlier, we can obtain the simulation results to verify our theoretical evaluation.

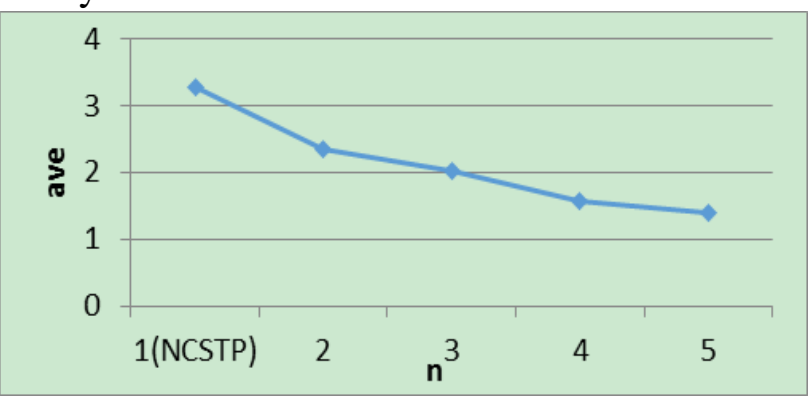

Fig. 6 Simulation result of PER $=0.1$

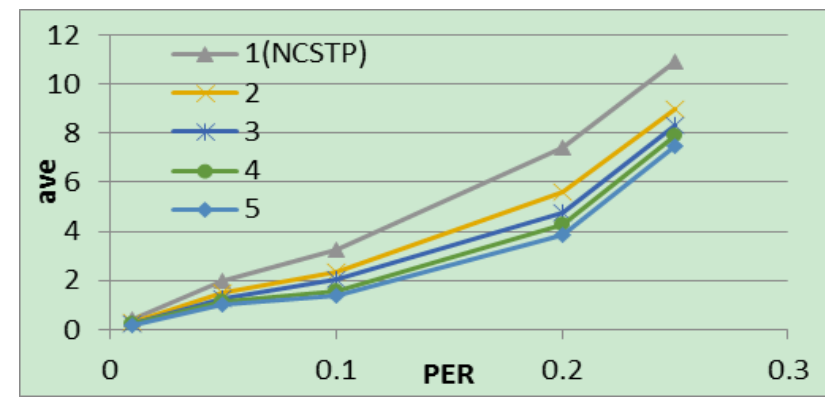

Fig. 7 Simulation result of different PERs

Results in Fig.6 reveal that the simulation results are approximately the same with theoretical evaluation. With our BCC mechanism (when $n>2$ ), the number of retransmission is smaller than NCSTP. The results shown in Fig.7 and 8 reveal that, the number of retransmission grows with PER and file size in both NCSTP and BCC. But in NCSTP, it grows much faster, which means that in high link error rate environment like deep space networks, BCC will achieve better performance than NCSTP in large file transmission. 


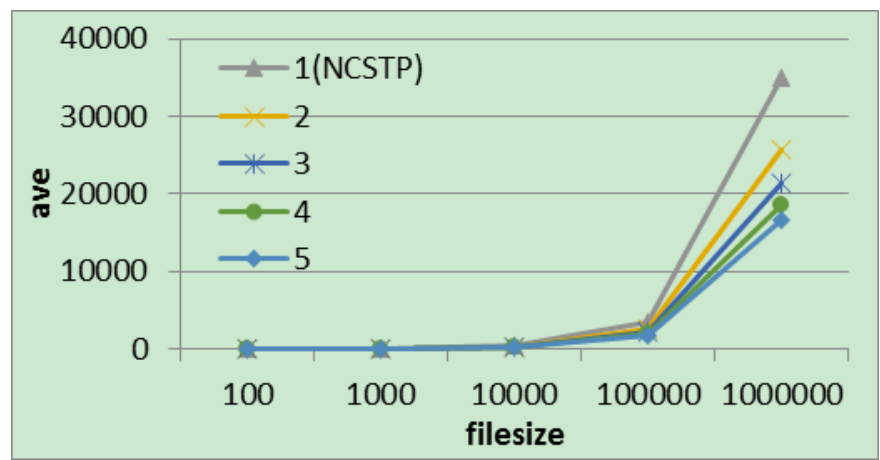

Fig. 8 Simulation result of different file sizes

\section{Conclusions and Future Works}

To solve the problems of burst packet loss which is caused by long propagation time and severe signal reduction in deep space networks, in this paper, we proposed block combination coding mechanism. Based on NCSTP, this mechanism can cope with burst packet loss through rational combination of several coded blocks without increasing the coding redundancy.

Through our theoretical and simulation evaluations, we can conclude that by combining the coded blocks in a reasonable manner, our BCC can effectively decrease the retransmission packets caused by burst packet loss. Due to the extreme long RTT in deep space links, a small amount of decrease in retransmission packets will cause a great reduction in transmission time.

In future works, we will consider the case of changing PERs under more practical scenarios and how to effectively deal with the link interruption.

\section{References}

[1] Fall K R. A delay-tolerant network architecture for challenged internets.[J]. SIGCOMM '03 Proceedings of the 2003 conference on Applications, technologies, architectures, and pro, 33(4):27-34 (2003)

[2] Cerf, V., Burleigh, S., Hooke, A., Torgerson, L., Durst, R., Scott, K., Weiss, H.: Delay tolerant networking architecture. RFC4838 (2007)

[3] Scott, K.L., Burleigh S.: Bundle protocol specification. J. RFC 5050 (2007)

[4] SHEETS, B. B. P.: CCSDS FILE DELIVERY PROTOCOL (CFDP). J. (2004)

[5] Ramadas, M., Burleigh, S.: Licklider transmission protocol-specification. J. RFC 5326, experimental (2008)

[6] Wood, L., Eddy, W.M., Ivancic, W., McKim, J., Jackson, C.: Saratoga: a Delay-Tolerant Networking convergence layer with efficient link utilization. In: Satellite and Space Communications. IWSSC'07. International Workshop, pp. 168-172. IEEE press (2007)

[7] Ioannis Psaras, Giorgos Papastergiou, Vassilis Tsaoussidis, Nestor Peccia: DS-TP: Deep Space Transport Protocol. In: Proceedings of IEEE Aerospace Conference, March, Montana, USA (2008)

[8] Papastergiou, G., Psaras, I., Tsaoussidis, V.: Deep-space transport protocol: a novel transport scheme for space DTNs. Computer Communications, 32(16), 1757-1767 (2009)

[9] Fu H, Yu W, Wu C, et al. NC-STP: A High Performance Network Coding Based Space Transport Protocol[M]//Wireless Algorithms, Systems, and Applications. Springer International Publishing, 34-43 (2014) 